\title{
Electron Diffraction and HRTEM Structure Analysis of Nanowires
}

\author{
José Reyes-Gasga, David Romeu and Alfredo Gómez-Rodríguez \\ Departamento de Materia Condensada, Instituto de Física, Universidad Nacional \\ Autónoma de México, Apartado postal 20-364, 01000,México D.F.
}

México

\section{Introduction}

In this work an electron diffraction analysis and an electron microscope structure study of nanowires are presented. Nanowires, as one-dimensionally nanostructured materials, have become the focus of intensive research due to their great potential for use as building blocks in the fabrication of electronic, optoelectronic, and sensor devices with nanoscale dimensions. Therefore, the importance of studying and understanding the electron diffraction phenomena and their implications over their electron microscopy images is great.

It has been indicated that the silver nanowires are highly faceted at nanometric scales. A comprehensive electron diffraction study on the structure of nanowires, firstly in a general approach and then in the specific case of silver nanowires, will be presented in this chapter. The important role of twinning in determining the habit of the final morphology will also be discussed.

One interesting feature of silver, gold, and copper pentagonal cross-section nanowires, is their remarkable structure (Gao et al., 2003; Giersig et al., 2004; Hofmeister et al., 2002; Sun et al., 2002; 2003; Zhao et al., 2005). It has been proposed that this structure evolved from a multi-twin decahedral nanoparticle growing in the [110] direction by stabilizing more effectively the newly formed 100 facets than the 111 facets and their electron diffraction patterns present "forbidden" spots.

The pentagonal arrangement in the multiple twinned particles (MTP) is quite known. MTP nanoparticles of transition metals with face-centered cubic (FCC) lattice (Heinemann et al., 1979; Howie \& Marks, 1984; Sun et al., 2002) have been reported. On the basis of these studies, the basic structure of a decahedral particle was described as the junction of five tetrahedral single crystals with twin-related adjoining faces along a common [110] edge in such a way that the [110] direction parallel to the five-fold axis is perpendicular to the $<110>$ direction of the edges, and that the normal vectors to all of them are parallel to $<100>$. The theoretical angle between two [111] planes is $70.5^{\circ}$, so by joining 5 tetrahedrons, which are bounded by 111 facets, a gap of $7.5^{\circ}$ is generated. Thus, to fill this gap some internal strain is necessary, giving place to dislocations and other structure defects (Heinemann et al., 1979; Howie \& Marks, 1984; Sun et al., 2002). These defects have been observed in the TEM cross-sectional images of the mentioned penta-twinned nanowires (Chen et al., 2004).

When observing nanowires with the high-resolution transmission electron microscope (HRTEM) very often we observe a contrast that can be interpreted at first sight as coming 
from some kind of an aperiodic array. Based on a five-twinned decahedron, it has been shown (Reyes-Gasga et al., 2006) that the HRTEM images of pentagonal cross-section nanowires can be interpreted as a moiré pattern contrast, and that their selected-area electron diffraction (SAED) patterns can be also completely generated through the same multi-twinned decahedron basis (Reyes-Gasga et al., 2006). However, up to today a completely satisfactory explanation of all the features present in the structure of these penta-twinned nanowires is lacking.

In this work the origin and structure analysis of the HRTEM contrast observed in nanowires will be also presented and it will be shown that they are closely related to a decahedron-base structure. To obtain the proper interpretation of the contrast presented by their HRTEM images, an understanding of the origin of these "forbidden" spots is necessary. We will, therefore, comment on the origin of these forbidden spots observed in the electron diffraction patterns of nanowires. The "forbidden" spots have been successfully indexed as corresponding to the first order Laue Zone (FOLZ) superimposed with the spots of the zero-order Laue zone (ZOLZ). Therefore, the HRTEM images presented a contrast produced by the interference of the zero-order Laue zone (ZOLZ) and FOLZ spots. The analysis of using of forbidden spots in the study of nanostructures has been successfully used in the interpretation of diffraction patterns of nanoparticles (Reyes-Gasga et al., 2008; Romeu \& Reyes-Gasga, 2002) and it is also presented.

In order to do a comprehensible reading of this chapter, we include, among other, the following topics:

- A brief introduction to the electron diffraction phenomenon, including the Ewald sphere and the Laue zones.

- Diffraction patterns and images of twinning structures.

- A brief introduction to HRTEM image formation.

- Changes in the electron diffraction as a function of the sample size.

- Diffraction patterns and HRTEM images of nanowires.

\section{Reciprocal lattices}

Given three vectors $\widehat{a}, \widehat{b}$ and $\widehat{c}$ that span a lattice, we can always obtain another set $\widehat{a}^{*}, \widehat{b}^{*}$ and $\widehat{c}^{*}$ (called "reciprocal vectors") and defined through:

$$
\begin{aligned}
& \widehat{a} \cdot \widehat{a}^{*}=\widehat{b} \cdot \widehat{b}^{*}=\widehat{c} \cdot \widehat{c}^{*}=1 \\
& \widehat{a} \cdot \widehat{b}^{*}=\widehat{a} \cdot \widehat{c}^{*}=\widehat{b} \cdot \widehat{c}^{*}=\widehat{b} \cdot \widehat{a}^{*}=\widehat{c} \cdot \widehat{a}^{*}=\widehat{c} \cdot \widehat{b}^{*}=0
\end{aligned}
$$

The reciprocal vectors thus defined have several properties among which we may list:

a) The three reciprocal vectors are linearly independent (i.e. they are not coplanar) and span another lattice, called "reciprocal lattice".

Both sets $\{\widehat{a}, \widehat{b}, \widehat{c}\}$ and $\left\{\widehat{a}^{*}, \widehat{b}^{*}, \widehat{c}^{*}\right\}$ are bases for the three-dimensional Euclidean space. Given an arbitrary vector $P$ there exist unique numbers $x, y, z, x^{\prime}, y^{\prime}$ and $z^{\prime}$ such that

$$
\begin{aligned}
& P=x \widehat{a}+y \widehat{b}+z \widehat{c} \\
& P=x^{\prime} \widehat{a}^{*}+y^{\prime} \widehat{b}^{*}+z^{\prime} \widehat{c}^{*}
\end{aligned}
$$


and, taking dot products, we can easily see that

$$
\begin{aligned}
P \cdot \widehat{a}^{*} & =x \\
P \cdot \widehat{b}^{*} & =y \\
P \cdot \widehat{c}^{*} & =z \\
P \cdot \widehat{a} & =x^{\prime} \\
P \cdot \widehat{b} & =y^{\prime} \\
P \cdot \widehat{c} & =z^{\prime}
\end{aligned}
$$

and

$$
\begin{aligned}
& P=\left(P \cdot \widehat{a}^{*}\right) \widehat{a}+\left(P \cdot \widehat{b}^{*}\right) \widehat{b}+\left(P \cdot \widehat{c}^{*}\right) \widehat{c} \\
& P=(P \cdot \widehat{a}) \widehat{a}^{*}+(P \cdot \widehat{b}) \widehat{b}^{*}+(P \cdot \widehat{c}) \widehat{c}^{*}
\end{aligned}
$$

b) the relationship between both sets of vectors can be cast in an explicit way:

$$
\begin{aligned}
\widehat{a}^{*} & =\frac{\widehat{b} \times \widehat{c}}{V} \\
\widehat{b}^{*} & =\frac{\widehat{c} \times \widehat{a}}{V} \\
\widehat{c}^{*} & =\frac{\widehat{a} \times \widehat{b}}{V}
\end{aligned}
$$

where

$$
V=\widehat{a} \cdot \widehat{b} \times \widehat{c}
$$

is the volume of the unit cell. In fact, an explicit evaluation shows that the volume $V^{*}$ of the reciprocal lattice unit cell is given by

$$
V^{*}=\widehat{a}^{*} \cdot \widehat{b}^{*} \times \widehat{c}^{*}=\frac{1}{V}
$$

c) consider a plane containing at least three non collinear lattice points (a "crystallographic plane"). Let $P, Q$ and $R$ be three such points, a normal to the plane is given by

$$
N=(Q-P) \times(R-P)
$$

and upon expanding we see that $N$ can be rewritten as

$$
N=V G
$$

for some $G$ in the reciprocal lattice. The conclusion is that the normal to a crystallographic plane can always be taken to be a reciprocal lattice vector.

Furthermore, this implies that the vector equation for a crystallographic plane has the form

$$
P \cdot G=z
$$

where $z$ is an integer. 
d) conversely, given a reciprocal lattice vector $G$ the plane

$$
P \cdot G=z
$$

(with integer $z$ ) is a crystallographic plane (with some further qualifications as discussed below).

As a matter of fact we have that if $P=h \widehat{a}+k \widehat{b}+l \widehat{c}$ and $G=u \widehat{a}^{*}+v \widehat{b}^{*}+w \widehat{c}^{*}$ the corresponding plane equation is given by

$$
h u+k v+l w=z
$$

and we know (from number theory) that such an equation has integer solutions if and only if $\operatorname{gcd}(u, v, w) \mid z$ (by gcd we mean the greatest common divisor). In particular, notice that in the specific case $z=1$ there will be solutions if and only if $\operatorname{gcd}(u, v, w)=1$ (the numbers $u, v$ and $w$ are relatively prime).

e) the Miller indices of the plane with normal $G$ can be calculated easily. The plane will intersect the $\widehat{a}$ axis whenever there is a real number $r$ such that $P=r \widehat{a}$ lies on the plane. Consequently $P \cdot G=r \widehat{a} \cdot G=z$ and

$$
r=\frac{z}{\widehat{a} \cdot G}
$$

(provided $\widehat{a} \cdot G \neq 0$, the case $\widehat{a} \cdot G=0$ will be considered later). Similarly, the intersections with the $\widehat{b}$ and $\widehat{c}$ axes will be given by vectors $Q=s \widehat{b}$ and $R=t \widehat{c}$ that satisfy the conditions

$$
\begin{aligned}
& s=\frac{z}{\widehat{b} \cdot G} \\
& t=\frac{z}{\widehat{c} \cdot G}
\end{aligned}
$$

The reciprocals of these intersections are

$$
\begin{aligned}
& \frac{1}{r}=\frac{\widehat{a} \cdot G}{z} \\
& \frac{1}{s}=\frac{\widehat{b} \cdot G}{z} \\
& \frac{1}{t}=\frac{\widehat{c} \cdot G}{z}
\end{aligned}
$$

equations that can be considered as valid even when $\widehat{a} \cdot G$ or $\widehat{b} \cdot G \widehat{c} \cdot G$ is zero.

But if $G=u \widehat{a}^{*}+v \widehat{b}^{*}+w \widehat{c}^{*}$, all this implies that

$$
\begin{aligned}
& \frac{1}{r}=\frac{u}{z} \\
& \frac{1}{s}=\frac{v}{z} \\
& \frac{1}{t}=\frac{w}{z}
\end{aligned}
$$

and the Miller indices of the plane are simply (uvw). 
A normal to the plane with indices $u, v, w$ is the vector $u \widehat{a}^{*}+v \widehat{b}^{*}+w \widehat{c}^{*}$, for this reason both the plane and the reciprocal lattice vector normal to the plane are labeled as ( $u v w)$. Conversely, given a reciprocal lattice vector $(u v w)$, it is normal to a family of planes with Miller indices that can be obtained from $u v$ and $w$ dividing them by their greatest common divisor (however, most of the time ones simply assumes this divisor to be 1).

$f$ ) the distance between two consecutive lattice planes with indices $u, v$ and $w$ is precisely

$$
d=|(u v w)|^{-1}
$$

(the reciprocal to the reciprocal lattice vector size). Bear in mind that $u, v$ and $w$ are coprime, otherwise it might appear that there are lattice planes arbitrarily close to each other.

In order to prove this important result consider planes

$$
P \cdot G=z
$$

where $G$ lies in the reciprocal lattice and $z$ is an integer. Assume $\operatorname{gcd}(u, v, w)=$,1 so $z$ can be any integer. Next, take the planes for which $z=0$ and $z=1$. The distance between these planes is the length of the vector parallel to $G$ going from the plane with $z=0$ to the plane with $z=1$. This vector will be of the form

$$
P=x G
$$

but since

$$
P \cdot G=1=x|G|^{2}
$$

we conclude that

$$
x=\frac{1}{|G|^{2}}
$$

and the distance is

$$
|P|=|x G|=\frac{|G|}{|G|^{2}}=\frac{1}{|G|}
$$

so the distance between planes $(u v w)$ is

$$
d=\frac{1}{|G|}
$$

where

$$
G=u \widehat{a}^{*}+v \widehat{b}^{*}+w \widehat{c}^{*}
$$

If $\operatorname{gcd}(u, v, w) \neq$,

$$
d=\frac{\operatorname{gcd}(u, v, w,)}{|G|}
$$

$g$ ) the lattice that is reciprocal to the reciprocal lattice is the original lattice.

$h$ ) the rational index law, known long before the discovery of x-ray diffraction, states that crystallographic planes always have rational indices.

i) the angle between two crystallographic planes is the angle between the reciprocal lattice vectors that are normal to the planes. This entails the so called law of the constancy of angles: in every crystal only certain angles between planes can be found, they correspond to low index reciprocal lattice vectors. 


\section{Diffraction}

The electrons leaving the electron gun are characterized by having very nearly the same energy $E$, which is typically of the order of some $100 \mathrm{kev}$. They travel forming a well collimated beam (beam divergences of the order of thousandths of radian) and the radiation may be very well represented as a plane wave of wavelength $\lambda$.

$\lambda$ and $E$ are related by

$$
E=\frac{h^{2}}{2 m \lambda^{2}}
$$

where $h$ is Planck's constant and $m$ is the electron mass. The momentum $\vec{p}$ of the electron is given as $h \vec{k}$ where $\vec{k}$ is a vector of size $|\vec{k}|=\frac{1}{\lambda}$ and has the same direction as the electron beam ( $\vec{k}$ is known as electron "wave vector"). From these relationships it follows that

$$
E=\frac{h^{2}|\vec{k}|^{2}}{2 m}
$$

For a given energy $E$, the possible values for $\vec{k}$ lie on a sphere of radius $\frac{1}{\lambda}$. This sphere is known as Ewald's sphere.

The sample under study is hit by the incoming electrons, some of them leave the sample unaltered (transmitted beam) whereas some are elastically dispersed (diffracted beams). In this work we assume that the sample is crystalline and that the sample-radiation interaction is elastic (i.e. that the electrons don't change their energy).

The detection system is formed by a photographic plate or an electron detector. The record of the radiation scattered in various directions forms the "diffraction pattern".

\subsection{The diffraction grating}

In order to understand the geometry of the diffraction phenomenon, first consider a simple one-dimensional row of identical atoms, figure 1.

Let $\widehat{a}$ be the vector generating this lattice. Consider neighboring atoms $B$ and $C$ and let the incident radiation have wave vector $\overrightarrow{k_{0}}$. Far from the row, as in elementary optics, what is observed depends on the phase difference between the rays leading to constructive or destructive interference. An observer far from the grating will see that the phase difference between rays from $B$ and $C$ is given by $\delta$ where

$$
\delta=|\vec{a}|\left(\cos (\alpha)-\cos \left(\alpha_{0}\right)\right)
$$

If we call $\vec{k}$ the wave vector after the grating, we have that

$$
\delta=\lambda\left(\vec{k}_{0}\right) \cdot \vec{a}
$$

and, since in order to obtain constructive interference we must have

$$
\delta=n \lambda
$$

(with $n$ being an integer) we have that the condition for constructive interference becomes

$$
\left(\vec{k}-\vec{k}_{0}\right) \cdot \vec{a}=n \lambda
$$




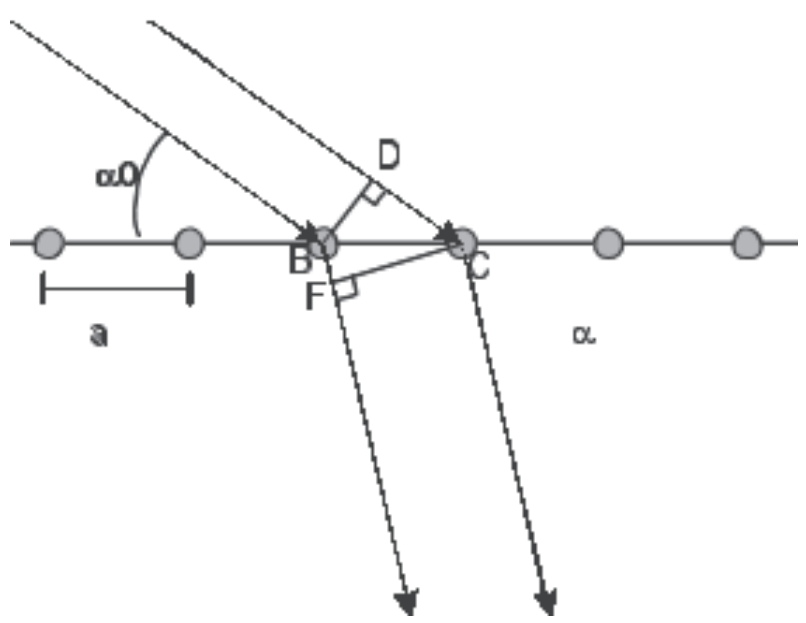

Fig. 1. Basic geometry of the one dimensional grid.

or

$$
\left(\vec{k}-\vec{k}_{0}\right) \cdot \vec{a}=n
$$

\subsection{The three-dimensional crystal}

A real three dimensional crystal can be thought of as a grid extending in three directions. Consequently if the crystal is generated by $\widehat{a}, \widehat{b}$ and $\widehat{c}$ the condition for constructive interference will be given by three equations similar to the previous one :

$$
\begin{aligned}
& \left(\vec{k}-\vec{k}_{0}\right) \cdot \vec{a}=m \\
& \left(\vec{k}-\vec{k}_{0}\right) \cdot \vec{b}=n \\
& \left(\vec{k}-\vec{k}_{0}\right) \cdot \vec{c}=p
\end{aligned}
$$

with $m, n$ and $p$ being integers. These equations are known as "Laue equations".

\subsection{The fundamental equation}

If we now call $\vec{g}=\left(\vec{k}-\vec{k}_{0}\right)$ and express this vector in terms of $\widehat{a}^{*}, \widehat{b}^{*}$ and $\widehat{c}^{*}$ we will have that

$$
\vec{g}=x \widehat{a}^{*}+y \widehat{b}^{*}+z \widehat{c}^{*}
$$

where $x, y$ and $z$ are three numbers to be determined. Multiplying this equation by $\widehat{a}, \widehat{b}$ and $\widehat{c}$ we have that

$$
\begin{aligned}
& x=\vec{g} \cdot \widehat{a} \\
& y=\vec{g} \cdot \widehat{b} \\
& z=\vec{g} \cdot \widehat{c}
\end{aligned}
$$

so (using Laue's equations)

$$
\vec{g}=m \widehat{a}^{*}+n \widehat{b}^{*}+p \widehat{c}^{*}
$$


The diffraction condition is that $\vec{g}$ be a reciprocal lattice vector.

\subsection{Ewald's construction}

The basic geometry for diffraction is shown in figure 2. Here we have indicated the incident

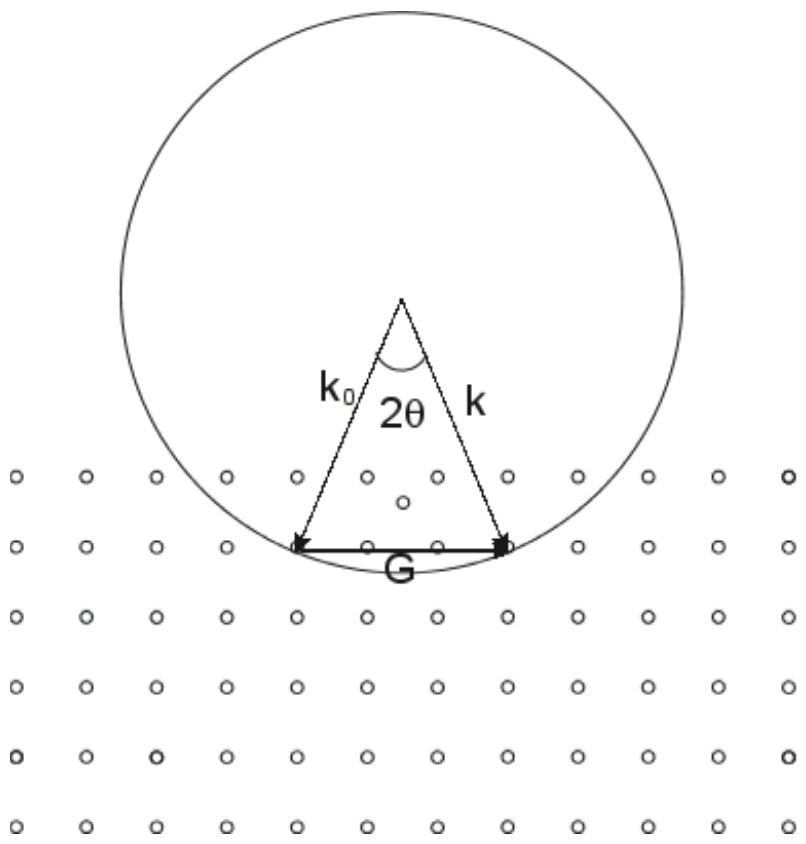

Fig. 2. Basic geometry for diffraction. The Ewald sphere is shown together with the diffraction condition.

and diffracted wave vectors ( $\widehat{k}_{0}$ and $\widehat{k}$ respectively), both are on Ewald's sphere as they correspond to electrons with the same energy. The tip of vector $\widehat{k}_{0}$ has been placed at the origin of the reciprocal lattice and the tip of $\widehat{k}$ has been placed over a point $\widehat{g}$ in the reciprocal lattice to better illustrate that $\widehat{k}-\widehat{k}_{0}$ is a reciprocal vector.

\subsection{Bragg's law}

There is a very simple way of visualizing the fundamental diffraction equation. In figure 3 we show schematically a family of planes that have a distance $d$ ("interplanar distance").

Call $\vec{g}$ the reciprocal lattice vector that is normal to these planes and, as we have already seen, it satisfies $d=|\vec{g}|^{-1}$. We also show (as arrows) the wave vectors $\vec{k}_{0}$ and $\vec{k}$ of the incident and diffracted waves. From the figure one can see that the angle $\theta$ between $\vec{k}_{0}$ and any plane is the same as the angle between $\vec{k}$ and the planes. This angle is called "Bragg angle". Since $\vec{g}=\left(\vec{k}-\vec{k}_{0}\right)$, taking the dot product of the equation with itself; we have that

$$
|\vec{k}|^{2}+\left|\vec{k}_{0}\right|^{2}-2 \vec{k} \cdot \vec{k}_{0}=|\vec{g}|^{2}
$$




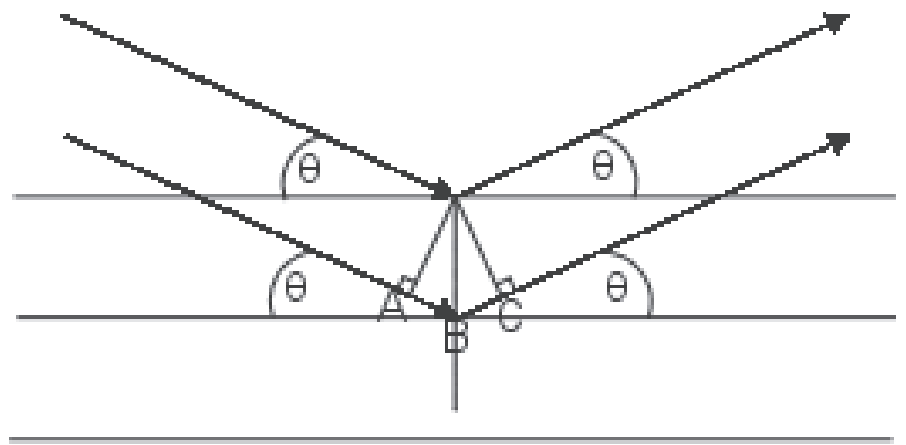

Fig. 3. Bragg's construction. The crystallographic planes behave as mirrors that reflect the radiation producing interference.

or

$$
2|\vec{k}|^{2}(1-\cos (2 \theta))=\frac{1}{d^{2}}
$$

where we have used the fact that the sizes of the incident and diffracted wave vectors are the same. Using a well known trigonometric identity for the cosine of the double of an angle we obtain

$$
2 d \operatorname{sen}(\theta)=\lambda
$$

Now, here we have used a $\vec{g}$ such that $d=|\vec{g}|^{-1}$ but in the fundamental equation $\vec{g}$ is any reciprocal lattice vector. For this reason we must modify the formula above to include all integer multiples of $\vec{g}$. Finally

$$
2 d \operatorname{sen}(\theta)=n \lambda
$$

and this is the famous Bragg equation. In geometrical terms we can say that the crystal behaves like a family of mirrors, there being one such a family for every set of parallel crystal planes. Radiation is reflected specularly by each family( that is, with equal incidence and reflection angles). The only difference with respect to ordinary mirrors is that the beams are reflected (diffracted) only when they arrive at the mirror forming the "right" angle, that is, Bragg's angle $\theta$.

\subsection{Approximating Ewald's sphere to a plane.}

In practice, for electrons, the radius of the sphere $\left(=\frac{1}{\lambda}\right)$ is very large compared to the reciprocal lattice vectors. Consequently it is possible to approximate the sphere to a plane in a neighborhood of the origin of reciprocal space. If the incident wave vector is $\vec{k}_{0}$ then the sphere resembles a plane with normal $\vec{k}_{0}$ and going through the origin of the reciprocal lattice. Ordinarily we don't give $\vec{k}_{0}$ directly but rather its direction $[h k l]$ in terms of the lattice. This vector is termed "zone axis".

It is important to remark that this approximation is not a good one for x-rays because in this case the radius of Ewald's sphere is comparable to the smallest reciprocal lattice vectors. 


\subsection{An example}

Consider an FCC crystal and assume the beam is along the [001]. A reciprocal lattice point (uvw) will contribute to the diffraction pattern if it satisfies

$$
[001] \cdot(u v w)=0
$$

The smallest vectors satisfying this equation and that also satisfying the condition that all of the indices are even or all of them are odd are (200) and (020) so the diffraction pattern will consist of all the integer combinations of these vectors.

\section{Higher order Laue zones}

For a given zone axis $[h k l]$ the planes

$$
[h k l](u v w)=0
$$

give, as indicated above, the diffraction pattern. But sometimes other reciprocal lattice planes also contribute to the pattern, and they are called "higher order Laue zones" (HOLZ) . They may arise as a result of having a very thin crystal or as a result of having an incomplete number of layers in a given crystallographic direction. The ordinary case $z=0$ is termed "zeroth order Laue zone" or ZOLZ.

Whatever the physical origin of these "forbidden spots", their geometry can be calculated for cubic crystals in two equivalent ways:

1. Calculate the various reciprocal lattice planes by means of

$$
[h k l](u v w)=n
$$

where $n$ is an integer. Then project the resulting points onto the zeroth-order Laue Zone $[h k l](u v w)=0$. Here $[h k l]$ is the direction of the incoming beam.

2. Calculate the lattice plane

$$
\left(u^{\prime} v^{\prime} w^{\prime}\right)\left[h^{\prime} k^{\prime} l^{\prime}\right]=0
$$

where now $\left(u^{\prime} v^{\prime} w^{\prime}\right)$ is a vector parallel to the incoming beam. Then calculate the (two-dimensional) lattice reciprocal to the lattice plane.

Both approaches give the same answer. The second method calculates a projection in reciprocal space that is reciprocal to a section in real space. Thus we have a particular instance of the well known section-projection theorem of Fourier theory.

In the previous equations we are using the convention that $[h k l]=k a+k b+l c$ where $a, b, c$ are lattice basis vectors and $(u v w)=u a^{*}+v b^{*}+w c^{*}$ where $a^{*}, b^{*}, c^{*}$ are the corresponding reciprocal lattice basis vectors.

However the second approach has several advantages:

- no need to solve for several values of $n$. In fact, all the values of $n$ are taken into account.

- No need to calculate projections at all, ZOLZ indices being automatically provided.

- Lends itself to the simple interpretation to be discussed below.

The practical procedure can be briefly summarized as: 
- If you know the beam is along the direction $[h k l]$ then determine the vector $\left(u^{\prime} v^{\prime} w^{\prime}\right)$ in the same direction (in the examples below this is very simple since a cubic lattice and its reciprocal lattice differ only in size).

- Determine basis vectors $a$ and $b$ for the section $\left(u^{\prime} v^{\prime} w^{\prime}\right)\left[h^{\prime} k^{\prime} l^{\prime}\right]=0$ (a Diophantine equation to be solved by standard methods).

- The reciprocal lattice vectors $a^{*}$ and $b^{*}$ can be determined from

$$
\begin{aligned}
& a^{*}=\frac{|b|^{2} a-(a \cdot b) b}{|a|^{2}|b|^{2}-(a \cdot b)^{2}} \\
& b^{*}=\frac{|a|^{2} b-(a \cdot b) a}{|a|^{2}|b|^{2}-(a \cdot b)^{2}}
\end{aligned}
$$

and all the integer combinations of them give all the allowed and forbidden spots.

\subsection{An example}

For instance, in the FCC (112) zone the equation to solve is

$$
(112)[h k l]=0
$$

whose solutions can be determined in many ways, possibly the easiest is by inspection so

$$
\begin{aligned}
& a=\frac{1}{2}[\overline{1} 10] \\
& b=[11 \overline{1}]
\end{aligned}
$$

and

$$
\begin{aligned}
& a^{*}=2 a=(1 \overline{1} 0) \\
& b^{*}=\frac{b}{3}=\frac{(11 \overline{1})}{3}
\end{aligned}
$$

(here we take advantage of the self reciprocity of cubic lattices). Notice that one of the points in the pattern is

$$
a^{*}-b^{*}=\frac{(2 \overline{4} 1)}{3}
$$

a "forbidden" spot actually being observed.

Diffraction spots (allowed or forbidden) are expected at

$$
g=m(1 \overline{1} 0)+\frac{n(11 \overline{1})}{3}
$$

with $m$ and $n$ being integers.

\section{Twinning}

A twin is an aggregation of several crystals of the same species whose relative orientations follow well-defined laws. These orientations are related by symmetry operations which do not belong to the crystal class of the untwinned crystal, either by a rotation about an axis 
$[u v w]$ or by reflection by a lattice plane $(h k l)$. The symmetry of the twin is generally higher than that of the untwinned crystal.

Two main kinds of twinning are distinguished, depending on whether the symmetry operation is: (a) $180^{\circ}$ rotation about an axis, called the twin axis, or (b) a reflection across a plane, called the twin plane.

\subsection{Mirrors}

Let $P$ be a vector and $P^{\prime}$ its image after reflection by a mirror that has $N$ as a normal. Then it can be easily shown that

$$
P^{\prime}=P-\frac{P \cdot N}{|N|^{2}} N
$$

For instance, in an FCC crystal, if $N=(111)$ and the crystal is $P=[100]$ oriented with respect to the incoming electron beam, the twin will be another crystal but oriented along $P^{\prime}=[100]-\frac{1}{3}[111]=\frac{1}{3}[2 \overline{1} \overline{1}]$ or, simply $[2 \overline{1} \overline{1}]$. In this way the diffraction patterns of the twins can be computed.

\section{Effect of incomplete layers}

Consider an FCC lattice. The smallest vectors in the (111) plane are

$$
\begin{aligned}
& a=\frac{1}{2}[1 \overline{1} 0] \\
& b=\frac{1}{2}[10 \overline{1}]
\end{aligned}
$$

(the $\frac{1}{2}$ factor come from the fact that the standard unit cell is not primitive) and the lattice reciprocal to the 2 dimensional lattice spanned by $a$ and $b$ is the one spanned by

$$
\begin{aligned}
& a^{*}=\frac{1}{2}[22 \overline{4}] \\
& b^{*}=\frac{1}{2}[2 \overline{4} 2]
\end{aligned}
$$

so "kinematically forbidden" spots will appear.

In the lattice there are, however, other (111) planes, they are stacked in the sequence $A B C A B C \ldots$. A vector taking the (111) layer $(A)$ to the next one $(B)$ is given by $c=[001]$, and a vector taking it to the third layer $(C)$ is $d=[002]$.

For this reason, the amplitude of a diffraction spot

$$
\mu=\alpha a^{*}+\beta b^{*}
$$

will be modulated by

$$
\begin{aligned}
\Psi & =1+\exp [2 \pi i(\mu \cdot c)]+\exp [2 \pi i(\mu \cdot d)] \\
& =1+\exp \left[2 \pi i\left(\frac{-4 \alpha+2 \beta}{3}\right)\right]+\exp \left[2 \pi i\left(\frac{-8 \alpha+4 \beta}{3}\right)\right]
\end{aligned}
$$


and the amplitude will be zero when

$$
-4 \alpha+2 \beta \equiv 1(\bmod 3)
$$

or

$$
-4 \alpha+2 \beta \equiv 2(\bmod 3)
$$

and these equations rule out the forbidden spots when the stacking is complete, in the sense that it comprises a whole $A B C A B C$...sequence.

\section{Direct and reciprocal spaces}

Any continuous function of space or time, no matter how complicated, can be expressed as a summation of a series of sine and cosine terms of increasing frequency. In other words, any continuous function of time or space can be described in terms of temporal or spatial frequencies, such as the number of cycles per unit time or the number of atomic planes per unit distance.

This duality in the possible representation of a function allows us to look at the physical property it describes in two different yet complementary spaces known as direct or physical and frequency spaces. In crystallography, these spaces are respectively given the names of real and reciprocal spaces. The latter has been described in detail in section 2 discussing the reciprocal lattice.

In order to gain some insight into the geometrical meaning of the Fourier transform, let us see how a simple sine wave $f(t)=\sin (2 \pi t)$ function is represented in both the direct and frequency spaces with the aid of figure 4 .
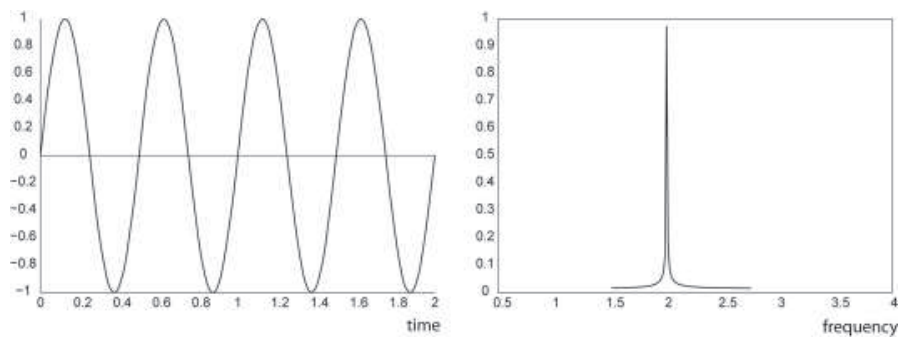

Fig. 4. A sine wave of unit amplitude and a frequency of $2 \mathrm{~Hz}$ represented in the time and frequency spaces.

On the left, fig. 4 shows 4 periods of the sine function with a frequency $v=2 \mathrm{~Hz}$, on the right the frequency space of the function, also called the power spectrum, is shown indicating that the function contains a single frequency of $2 \mathrm{~Hz}$. We now add two more waves, one with a third of the amplitude of the original and a frequency 3 times larger (3rd harmonic) and another with an amplitude $1 / 5$ of the original and a frequency 5 times larger ( 5 th harmonic):

$$
f(t)=\sin (2 \pi v t)+\frac{1}{3}(\sin (2 \pi 3 v t))+\frac{1}{5}(\sin (2 \pi 5 v t))
$$

and the time and frequency representations are shown in figure 5 . Note that the shape of the new function no longer resembles a sine wave.

If we include the first 20 odd harmonics, a square wave pattern starts to emerge as shown in figure 6 . This simple calculation already shows that in order to resolve rapid variations of a 

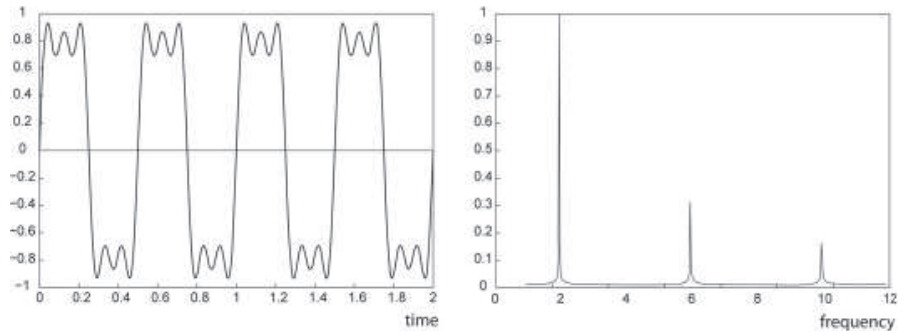

Fig. 5. The time and frequency space representations of a $2 \mathrm{~Hz}$ sine wave and its first two odd harmonics (equation 81).

function, such as the sharp edges of a square wave, we need higher frequencies. The higher the cut-off frequency, the better the approximation.
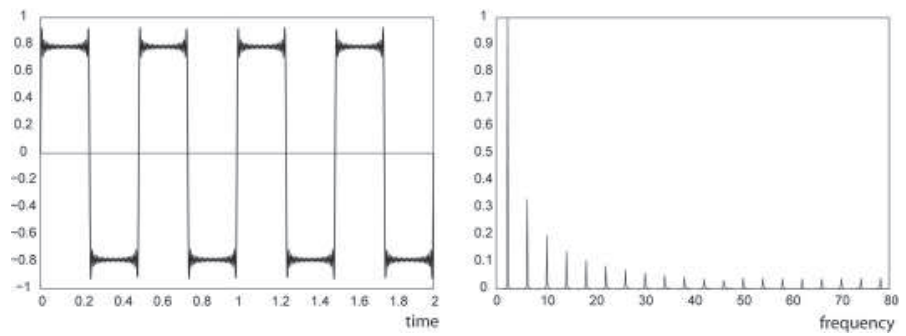

Fig. 6. The time and frequency space representations of the first 20 odd harmonics of a $2 \mathrm{~Hz}$ sine wave.

Since the sine is an odd function, i.e. $\sin (a)=-\sin (-a)$, only odd functions can be reproduced by combinations of them. We could use cosines instead of sines in the expansion, but being even functions, i.e., $\cos (a)=\cos (-a)$, they allow only the reproduction of even functions. Since most functions are asymmetric (neither odd nor even) a combination of both sines and cosines is needed to reproduce arbitrary functions. As a result, a general function of time (or space) in one dimension can be expressed as

$$
f(t)=a_{0}+a_{1} \cos (\omega t)+a_{2} \cos (2 \omega t)+\ldots+b_{1} \sin (\omega t)+b_{2} \sin (2 \omega t)+\ldots
$$

or, more succinctly:

$$
f(t)=\sum_{i=0}^{\infty} a_{i} \cos (i \omega t)+\sum_{i=0}^{\infty} b_{i} \sin (i \omega t)
$$

where $\omega=2 \pi v$ and $a_{i}, b_{i}$ are constants. In general, any non-periodic continuous function $f(x)$ of a single variable $x$ can be expanded as

$$
f(x)=\sum_{i=0}^{\infty} F(u) e^{2 \pi i x u}
$$

where $u$ is the temporal or spatial frequency variable and $F(u)$ is given by

$$
F(u)=\int_{-\infty}^{\infty} f(x) e^{-2 \pi i x u} d x
$$


In general, $F(u)$ will be a complex quantity even though the original data are purely real. The meaning of this is that not only the amplitude of each frequency present is important, but also its phase relationship to the others.

Equation 84 is known as the Fourier transform of the function $f(x)$ and equation 85 is the inverse transformation which is very similar except for the sign of the exponential term.

The time (or space) and frequency representations of a function are equivalent in the sense that besides size effects, they convey the same amount of information although they have very different meanings. For our purposes, the most important property of the Fourier transform is that it relates the direct or physical space containing a crystal lattice and the reciprocal space containing the reciprocal lattice and the kinematical diffraction pattern (see section 3 ).

For an infinite crystal, the direct and reciprocal lattices are given in terms of each other by the three dimensional form of equations 84 and 85 . Since the size of real samples is always finite, this leads to important size and shape effects which become especially conspicuous in the case of nanoparticles. In what follows we shall briefly describe what these effects are in order to be able to properly interpret a micro-diffraction pattern

\section{Size and shape effects}

We have already seen that the higher the frequencies in Fourier (diffraction) space, the more detailed is the pattern in the physical space, the reason being that sharper features require smaller wavelengths to be reproduced. We shall further illustrate this property with the aid of fig. 7 which also serves to illustrate the effect the specimen shape has on its diffraction pattern. Figure 7 shows the Fourier transform of a function which equals one inside the black triangular region shown on the left hand side and zero elsewhere. Note that the Fourier transform has large spikes in the directions normal to the edges of the triangular region corresponding to the high frequency components needed to reproduce the sharp changes of the function at the boundaries of the triangle.
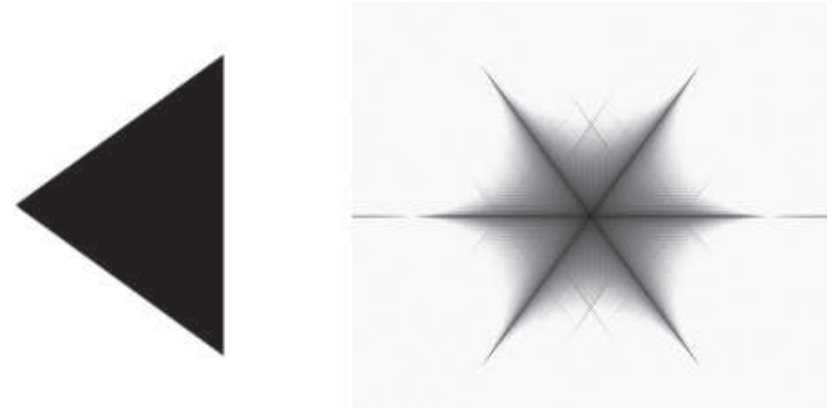

Fig. 7. Left: a triangular shape function which equals one inside the black triangle and zero outside. Right: its Fourier transform rescaled for clarity.

The size of the specimen modifies the size of the diffraction pattern spots. The easiest way to illustrate this is by calculating the Fourier transform of two waveforms of different length. Figure 8 shows the Fourier transform of the same function used in figure 5 (eq. 81) but using a different number of periods. Note that as the length of the waveform representing the size of a crystal increases, the width of the spectral components decreases. In the limit, for an infinite wave, the Fourier peaks become Dirac delta functions. 

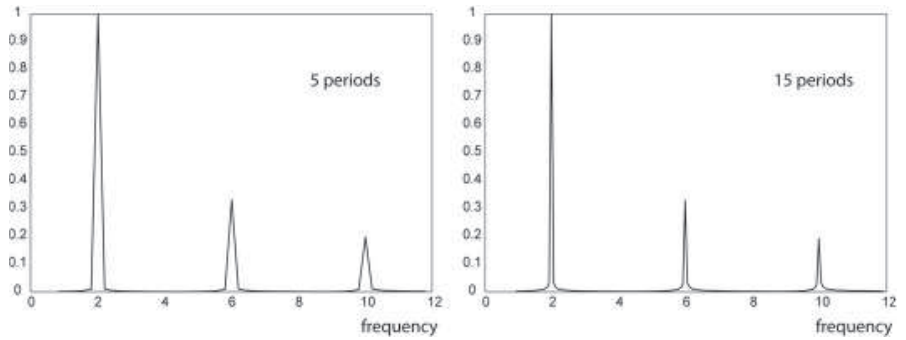

Fig. 8. Fourier transform of 5 and 15 periods of the function defined by equation 81

This effect is further illustrated in figure 9 which shows the kinematical diffraction pattern of two FCC nanoparticles of two different sizes. Note the spot size is smaller in the case of the larger particle and the diffraction lines which are normal to the faces of the nanoparticle.

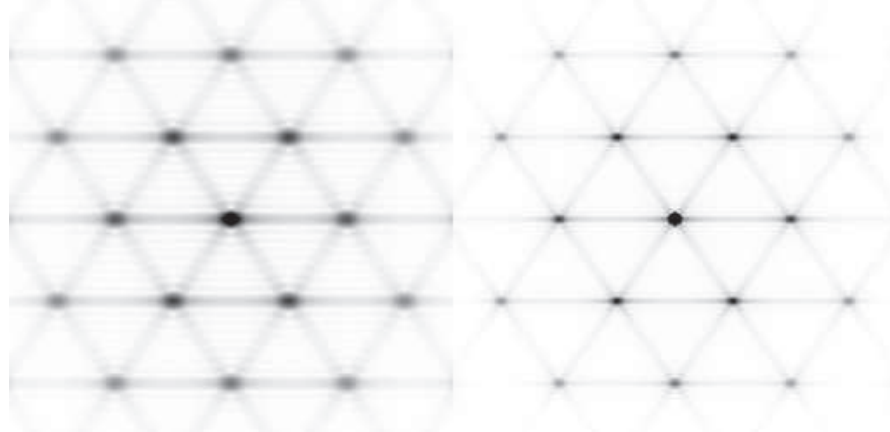

Fig. 9. Kinematical diffraction pattern (Fourier Transform) taken along the [011] direction) of two FCC nanoparticles composed of: left: 255 atoms and right: 1785 atoms.

\section{The convolution theorem}

The mathematical way to express the aforementioned size and shape effects is known as the convolution theorem. Succinctly, the theorem states that the Fourier transform of the product of two functions is equal to the mathematical convolution of their Fourier transforms. In other words, if $f(x)$ and $g(x)$ are integrable functions with Fourier transforms $F(u)$ and $G(u)$ respectively, then the Fourier transform of the product (denoted $\widehat{f g}(u)$ ) is given by the convolution (denoted by $*$ ) of the individual Fourier transforms.

$$
\widehat{f g}(u)=F(u) * G(u)
$$

The geometrical meaning of the convolution is simpler to express graphically. Figure 10 shows schematically the convolution of two graphic functions.

Since any nanoparticle of arbitrary size and shape can always be considered as an infinite crystal chopped by a "shape" function valued one inside the nanoparticle's volume and zero elsewhere, it turns out that its kinematical diffraction pattern will be the convolution of the Fourier transform of the crystal (the reciprocal lattice) with the Fourier transform of the shape. This is illustrated in fig.10 whose top three figures show from left to right the real space 


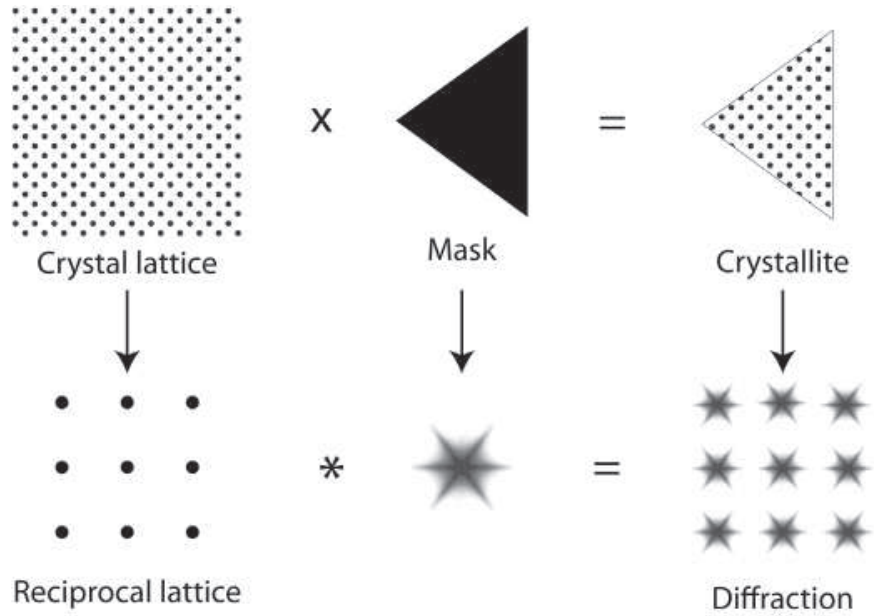

Fig. 10. Convolution of a set of reciprocal lattice points the Fourier transform of the triangular shape shown in fig. 7, representing schematically the reciprocal lattice of a small triangular bi-dimensional nanoparticle.

representation of a section of an infinite 2D crystal lattice, the masking function and the crystallite resulting from their multiplication. The corresponding Fourier transform i.e. the reciprocal space representation of each figure is shown below. Note that the effect of the convolution is to "stamp" each reciprocal lattice point with a copy of the Fourier transform of the shape function.

\section{A brief introduction to HRTEM formation}

The image formation in a conventional TEM and HRTEM microscope includes the microscope itself, the sample under study, the orientation relationship between the electron beam and the sample, and the objective aperture of the microscope. The contrast observed in the screen of the microscope depends on the aperture size and the diffraction (or reciprocal space) spot(s) going through it, and of course on the quality of the microscope. Thus, if the transmitted beam is the only one going through the objective aperture, we will observe the amplitude contrast in bright field mode (Fig. 11). In the case of a diffracted beam, a dark field image is observed. With more than one reflection, the quality of the microscope plays the main role: the "line" resolution requires that two beams go through the objective aperture, and the HRTEM requires the greatest number of reflections (Fig. 12). The most important point we must have in mind is that any reflection passing through the objective aperture is going to be included in the contrast of the obtained image, and once the image obtained any change can be done regarding the information of the sample under study. Figure 13 shows the HRTEM image of one of the particles shown in figure $12 \mathrm{C}$. The insets show the periodicity of the image by the FFT (Fast Fourier Transform) spectra calculated with the Digital Micrograph software.

\section{Pentagonal silver nanowires}

The synthesis for silver nanowires has been reported elsewhere (Brust et al., 1995). Silver nanowires were synthesized by the polyol reduction of silver nitrate (AgNO3) in presence 

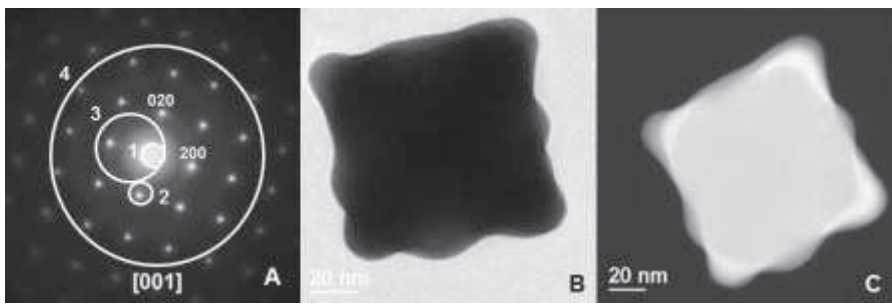

Fig. 11. A) Schematic representation of the size and position of the objective aperture in the focal plane of the objective lens for image formation in a TEM in different situations: bright field image (1), dark field image (2), line-resolution image (3) and high-resolution image (4). B) Bright field and C) dark field image of an Au particle.

of PVP. Scanning electron microscopy of the nanowires was conducted using the scanning electron microscope (SEM) Hitachi 4500F. Transmission electron microscopy was conducted in a HRTEM JEOL 2010F microscope equipped with Schottky-type field emission gun, ultra-high resolution pole piece $(C s=0.5 \mathrm{~mm})$, and a scanning transmission electron microscope (STEM) unit with high angle annular dark field (HAADF) detector operating at $200 \mathrm{kV}$. For digital image processing the Digital Micrograph (GATAN) software was used. To simulate the electron diffraction patterns, the SimulaTEM software (Gomez et al., 2010) was employed. Figure 14 shows the SEM images of the synthesized Ag nanowires where their pentagonal cross-section is presented.

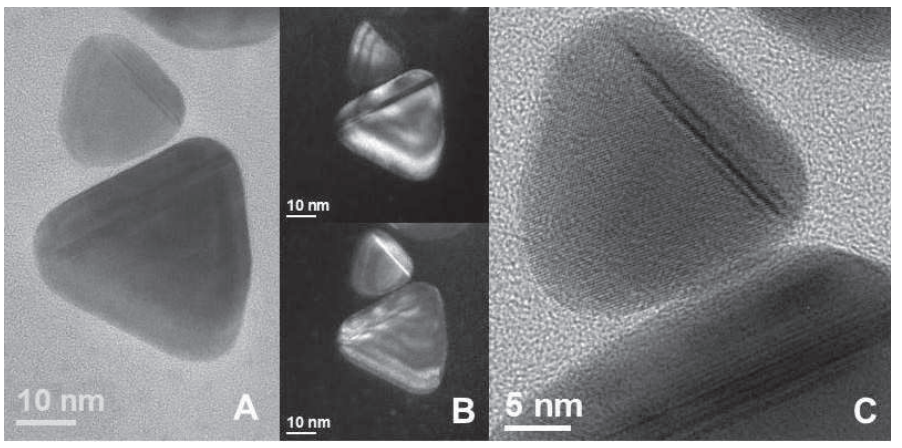

Fig. 12. Example of a bright field image (A), two dark field images using a different reflection in each case (B), and a high-resolution image (C) of Au nanoparticles.

\section{Diffraction patterns and HRTEM images of pentagonal silver nanowires}

Once in the HRTEM microscope, conventional bright field (Fig. 15A) and HRTEM (Fig. 15B) images and electron diffraction patterns of an Ag nanowire are obtained easily. Figure 16 shows the electron diffraction patterns of an individual pentagonal Ag nanowire when the electron beam goes through the directions indicated by (A) and (B) in the drawings, respectively. Because the pentagonal rotational periodicity, there is an angle of $18^{\circ}$ between them. The analysis and indexation of these diffraction patterns indicate that they correspond to the overlapping of the [001] and [112] zone axes (Fig. 16A), and of the [111] and [110] zone 


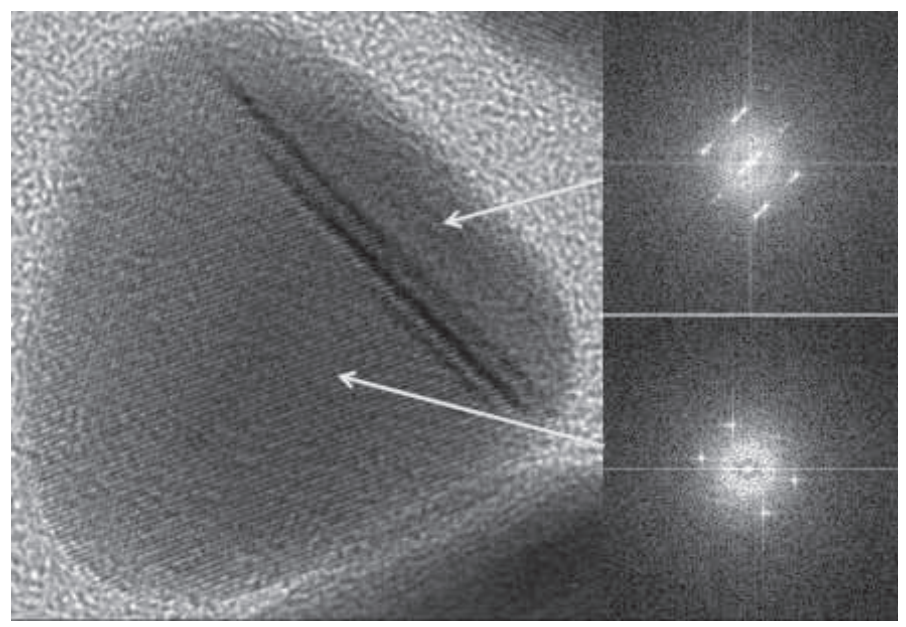

Fig. 13. Magnification of the high-resolution image shown in figure $2 \mathrm{C}$. The insets show the FFT spectra calculated with the Digital Micrograph software where the periodicity obtained en each region of the Au nanoparticle is shown.

axes (Fig. 6B), of the silver FCC unit cell, respectively. The presence of an aperiodic sequence of diffraction spots, mainly in figure $16 \mathrm{~A}$ is of notorious relevance.

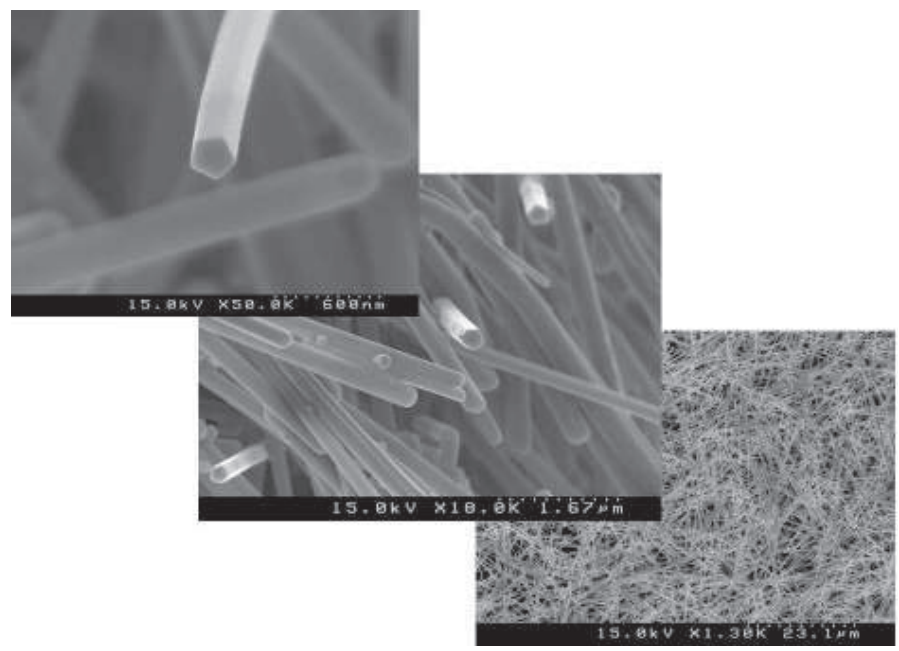

Fig. 14. SEM images of the synthesized Ag nanowires showing their pentagonal cross-section.

In order to understand the contrast observed in the HRTEM images of the pentagonal Ag nanowires is quite important to know their structure. A HRTEM image from the area close to the tip is shown in figure 17. The upper right inset shows the FFT from the indicated squared contrast, which corresponds to the [001] zone axis. The lower right inset shows the FFT from the region enclosed by the square in the HRTEM image. The analysis of this FFT indicates that 


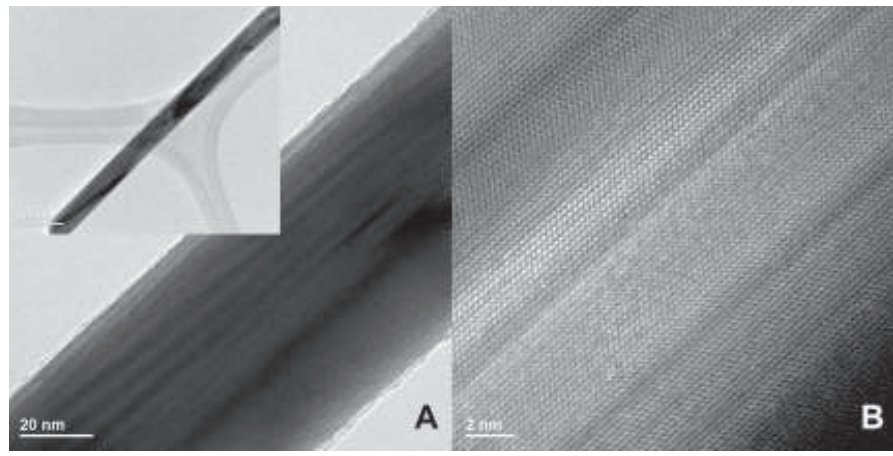

Fig. 15. TEM (A) and HRTEM (B) images of an Ag nanowire in its central region. The inset shows the TEM bright-field image of the nanowire at lower magnification.

it corresponds to the same overlapping diffraction pattern calculated in figure 16A, including the aperiodic sequence.
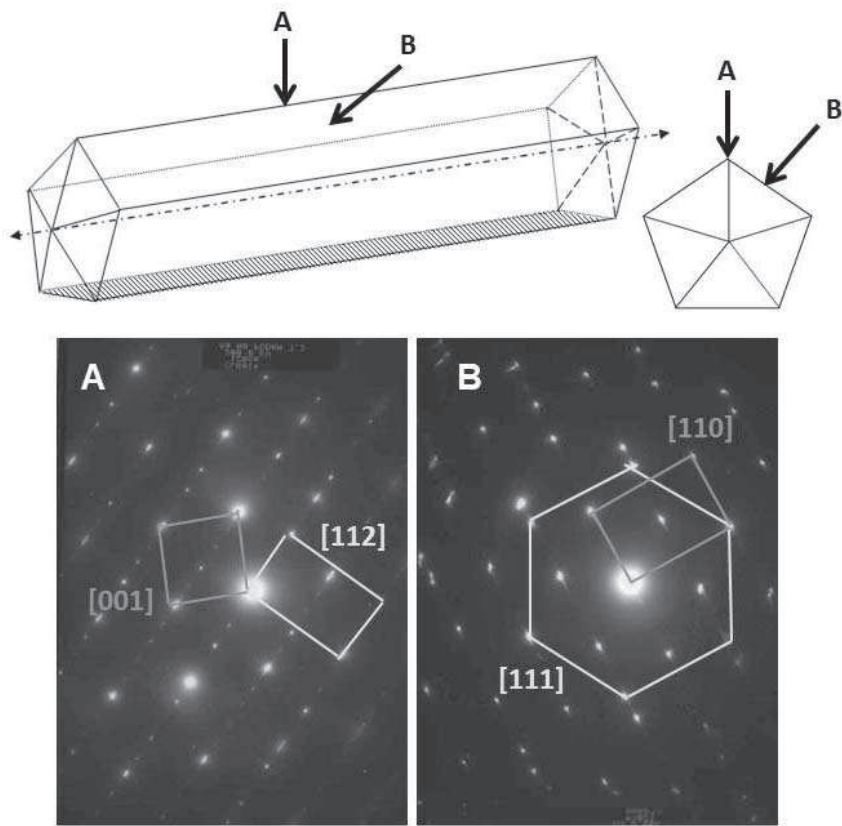

Fig. 16. Characteristic diffraction patterns of a pentagonal Ag nanowire when the electron beam goes through the directions indicated by (A) and (B) in the drawings, respectively. Their indexation indicates that they correspond to the overlapping of the [001] and [112] zone axes (A), and of the [111] and [110] zone axes (B). Note the existence of an aperiodic sequence of diffraction spots in (A). 


\section{Interpretation of the reflections observed in the diffraction patterns of pentagonal silver nanowires}

Let us show that the observation of this aperiodic sequence of diffraction spots is produced by the nano-size-dimension of the nanowires in combination with double diffraction due to twinning. The nanometric size of the nanowire along its cross-section produces the enlargement of reciprocal spots, then it is easily deduced that some of the spots composing the diffraction pattern shown in figure 16 are in fact spots of not only to the Zero Order Laue Zone (ZOLZ), but also to the First Order Laue Zone (FOLZ). Schematic drawings of the fcc reciprocal space including the ZOLZ and the "upper" and "lower" FOLZ spots for both [001] and [112] zone axes and for both [110] and [111] zone axes are shown in figures 18 and 19, respectively. As the FOLZ spots are elongated, they intercept the Ewald sphere. The drawings in the corresponding squares of these figures show the generated pattern when the diffraction patterns, including their respective FOLZ spots, overlap according to the orientation relationships indicated in the diffraction patterns of figure 16. Note that many of the diffracted spots shown in figure 16 are reproduced.

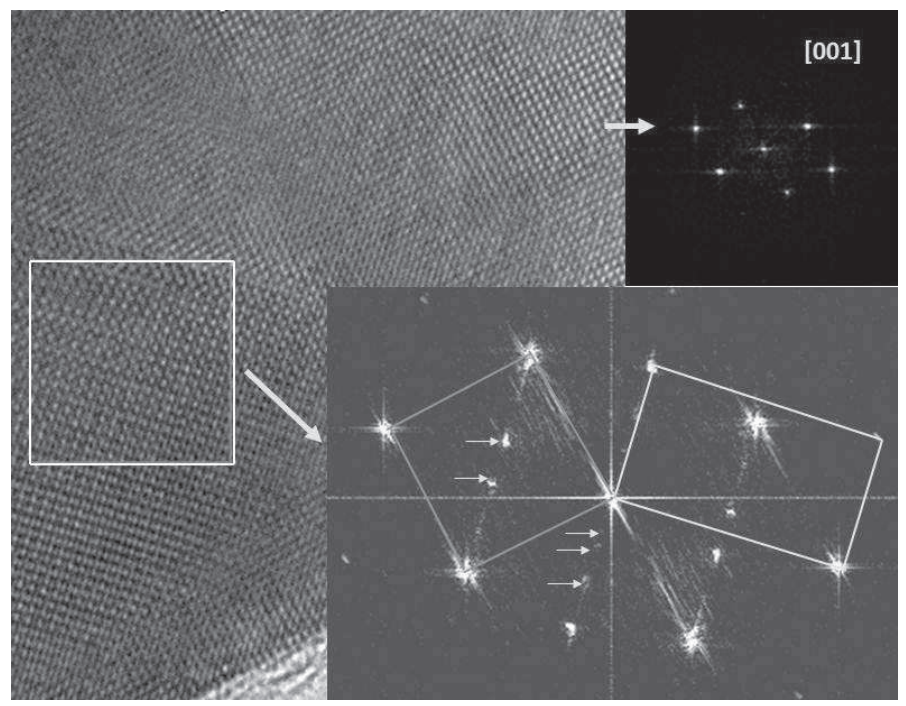

Fig. 17. HRTEM image from an area close to the tip of a pentagonal Ag nanowire. The upper right inset shows the FFT from the squared contrast; the lower right inset shows the FFT from the region enclosed by a square. Note the existence of the same spots as those in the diffraction pattern of figure 16A.

\section{Final remarks}

Reyes-Gasga et al. (Reyes-Gasga et al., 2006) have already proposed the structure of the Dh-NWs as a chain of decahedra joined along the vertex (which is parallel to the 5-fold symmetry). In this model, the Dh-NWs will be always growing along the [111] direction. Figure 20 shows the comparison among the experimental diffraction patterns shown in figure $16(\mathrm{~A})$ and $(\mathrm{B})$ and the simulated diffraction patterns from an $\mathrm{Ag}$ decahedral (Ad and 


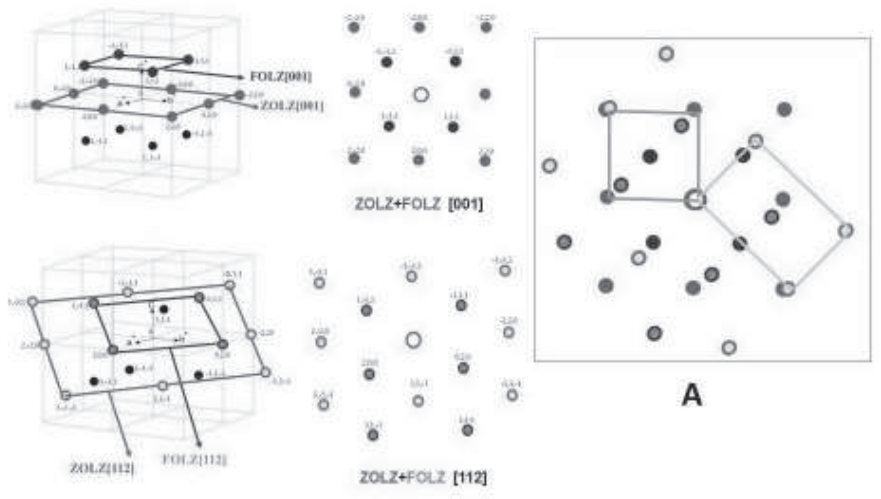

Fig. 18. . Schematic drawing of the fcc reciprocal space along the [001] and [112] zone axes including the ZOLZ and the "upper" and "lower" FOLZ spots. The patterns have been indexed accordingly. The square (A) shows the generated pattern when these patterns overlap according to the orientation relationships indicated in figure $16 \mathrm{~A}$.

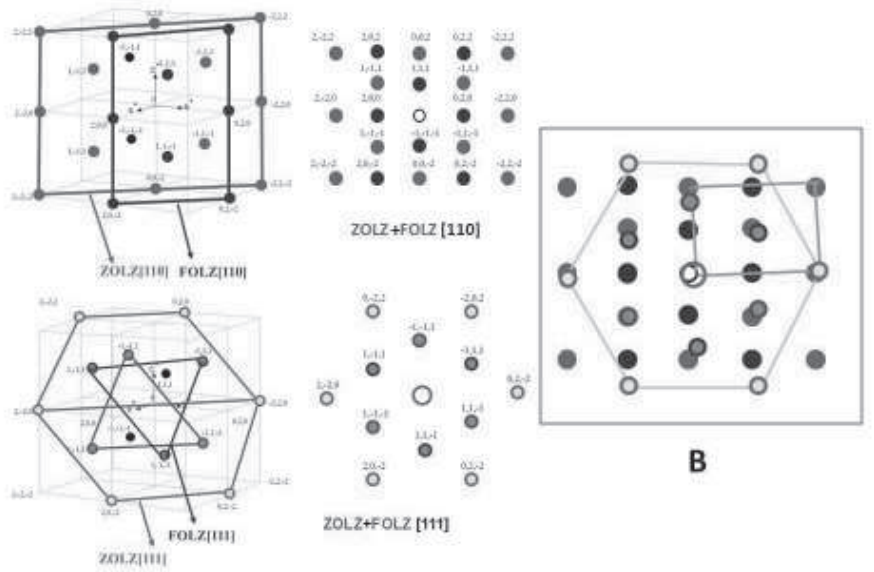

Fig. 19. Schematic drawing of the fcc reciprocal space along the [110] and [111] zone axes including the ZOLZ and the "upper" and "lower" FOLZ spots. The patterns have been indexed accordingly. The square (B) shows the generated pattern when these patterns overlap according to the orientation relationships indicated in figure 16B.

Bd) and an Ag elongated decahedron (Aed)) and (Bed). Note that the aperiodic sequence observed in the experimental patterns is reproduced in the case of the decahedron, while the elongated model generates a different aperiodic sequence. This idea of the chain of decahedra joined along the vertex has been successfully used in the analysis and interpretation of diffraction patterns of Au nanoparticles (Reyes-Gasga et al., 2008; Romeu \& Reyes-Gasga, 2002). However, observing the simulated patterns shown in figure 20 , we conclude that there is still a lot of work to be done in this direction. 

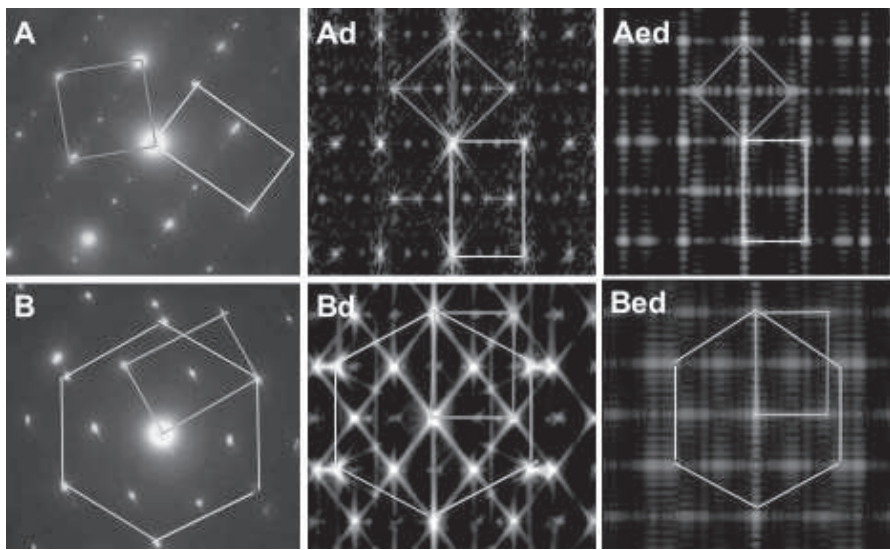

Fig. 20. Comparison among the experimental diffraction patterns shown in figure 16 (A) and (B) and the simulated diffraction patterns from an $\mathrm{Ag}$ decahedral (Ad and $\mathrm{Bd}$ ) and an $\mathrm{Ag}$ elongated decahedron (Aed and Bed).

\section{Acknowledgements}

We are grateful to Prof. Dr. M. Jose Yacaman, Dr. J. L. Elechiguerra, and Dr. Xiaoxia Gao for supplying the samples used in this work and for their collaboration as coauthors of joint papers. We also thank S. Tehuacanero-Nuñez, P. Mexia-Hernández, R. Hernandez-Reyes, J. Cañetas and M. Monroy-Escamilla for their technical support in the elaboration of this work.

\section{References}

Brust, M.; Fink, J.; Bethell, D.; Schiffring D. J. \& Kiely, C. (1995). Synthesis and reactions of functionalized gold nanoparticles. J. Chem. Soc. Chem. Comm. , 1655, Issue 16. DOI: 10.1039/C39950001655.

Chen, H.; Gao, Y.; Zhang, H.; Liu, L.; Yu, H.; Tian, H.; Xie, S. \& Li, J. (2004). Transmission-electron-microscopy study on fivefold twinned silver nanorods. J. Phys. Chem. B., Vol.108, No. 32, (August 2004), pp 12038-12043.

Gao, Y.; Jiang, P.; Lui, D. F.; Yuan, H. J.; Yan, X. Q.; Zhou, Z. P.; Wang, J. X.; Song, L.; Liu, L. F.; Zhou, W. Y.; Wang, G.; Wang, C. Y. \& Xie, S. (2003). Synthesis, characterization and self-assembly of silver nanowires. Chem. Phys. Lett., Vol.380, No. 1-2, (October 2003), pp 146-149.

Giersig, M.; Pastoriza-Santos, I.; \& Liz-Marzán L. M. (2004). Evidence of an aggregative mechanism during the formation of silver nanowires in N,N-dimethylformamide $J$. Mater. Chem., Vol.14, No. 4 (April 2004), pp 607-611.

Heinemann, K.; Yacamán, M. J.; Yang, C. Y. \& Poppa, H. (1979). The structure of small, vapor-deposited particles: I. Experimental study of single crystals and particles with pentagonal profiles. J. Crystal Growth, Vol.47, No. 2, (August 1979), pp 177-186.

Hofmeister, H.; Nepijko, S. A.; Levlev, N. N.; Schulze, W.; \& Ertl. G. (2002). Composition and lattice structure of fivefold twinned nanorods of silver. J. Crystal Growth, Vol.234, No. 4, (February 2002), pp 773-781.

Howie A. \& Marks, L. D. (1984). Elastic strains and energy balance for multiply twinned particles Phil. Mag., A Vol.49, No. 1 (January 1984), pp 95-109. 
Reyes-Gasga, J.; Elechiguerra, J. L.; Liu, C.; Camacho-Bragado, A.; Montejano-Carrizales, J. M. \& Yacamán, M. J. (2006). On the structure of nanorods and nanowires with pentagonal cross-sections. J. Cryst. Growth, Vol.286, No. 1, (January 2006), pp 162-172.

Reyes-Gasga, J.; Gómez-Rodríguez, A.; Gao, X. \& José-Yacamán, M. (2008). Ultramicroscopy, Vol.108, No. 9, (August 2008), pp 929-936

Romeu, L.D. \& Reyes-Gasga, J. (2011). Interpretation of the nano-electron-diffraction patterns along the five-fold axis of decahedral gold nanoparticles. Microc.Microanal, Vol.17, in press. DOI 10.1017/s1431927610094511.

Sun, Y.; Gates, B.; Mayers, B. \& Xia, Y. (2002). Crystalline silver nanowires by soft solution processing. Nanoletters, Vol.2, No. 2, (February 2002), pp 165-168.

Sun, Y.; Mayers, B.; Herricks T. \& Xia, Y. (2003). Polyol synthesis of uniform silver nanowires: a plausible growth mechanism and the supporting evidence. Nanoletters, Vol.3, No. 7, (July 2003), pp 955-960.

Zhao, Q.; Qiu, J.; Zhao, C.; Hou L. \& Zhu, C. (2005). Synthesis and formation mechanism of silver nanowires by a templateless and seedless method Chem. Letters, Vol.34, No. 1, (January 2005), pp 30-31. 


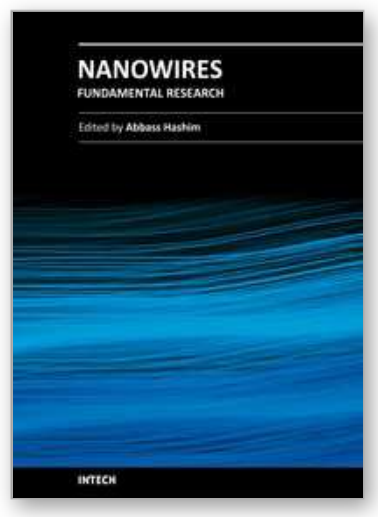

\author{
Nanowires - Fundamental Research \\ Edited by Dr. Abbass Hashim
}

ISBN 978-953-307-327-9

Hard cover, 552 pages

Publisher InTech

Published online 19, July, 2011

Published in print edition July, 2011

Understanding and building up the foundation of nanowire concept is a high requirement and a bridge to new technologies. Any attempt in such direction is considered as one step forward in the challenge of advanced nanotechnology. In the last few years, InTech scientific publisher has been taking the initiative of helping worldwide scientists to share and improve the methods and the nanowire technology. This book is one of InTechâ $€^{\mathrm{TM}_{\mathrm{S}}}$ attempts to contribute to the promotion of this technology.

\title{
How to reference
}

In order to correctly reference this scholarly work, feel free to copy and paste the following:

David Romeu, Alfredo Gomez and Jose Reyes-Gasga (2011). Electron Diffraction and HRTEM Structure Analysis of Nanowires, Nanowires - Fundamental Research, Dr. Abbass Hashim (Ed.), ISBN: 978-953-307327-9, InTech, Available from: http://www.intechopen.com/books/nanowires-fundamental-research/electrondiffraction-and-hrtem-structure-analysis-of-nanowires

\section{INTECH}

open science | open minds

\section{InTech Europe}

University Campus STeP Ri

Slavka Krautzeka 83/A

51000 Rijeka, Croatia

Phone: +385 (51) 770447

Fax: +385 (51) 686166

www.intechopen.com

\section{InTech China}

Unit 405, Office Block, Hotel Equatorial Shanghai

No.65, Yan An Road (West), Shanghai, 200040, China

中国上海市延安西路65号上海国际贵都大饭店办公楼405单元

Phone: +86-21-62489820

Fax: +86-21-62489821 
(C) 2011 The Author(s). Licensee IntechOpen. This chapter is distributed under the terms of the Creative Commons Attribution-NonCommercialShareAlike-3.0 License, which permits use, distribution and reproduction for non-commercial purposes, provided the original is properly cited and derivative works building on this content are distributed under the same license. 